\title{
Dieta y cáncer de mama en Latinoamérica
}

\author{
Luisa Torres-Sánchez, DSP,(1) Marcia Galván-Portillo, DrSP,(') Sarah Lewis, MSc, (2) \\ Héctor Gómez-Dantés, MD, M en C, (2) Lizbeth López-Carrillo, PhD.(I)
}

Torres-Sánchez L, Galván-Portillo M, Lewis S, Gómez-Dantés H, López-Carrillo L.

Dieta y cáncer de mama en Latinoamérica. Salud Publica Mex 2009;5 I supl 2:SI8I-SI 90.

\section{Resumen}

Mundialmente el cáncer de mama (CM) es la primera causa de muerte por tumores malignos entre las mujeres. Objetivo. Revisar los estudios epidemiológicos más relevantes sobre dieta y CM en América Latina y el Caribe (ALC). Material y métodos. La búsqueda sistemática realizada en México en el lapso de junio a noviembre del año 2008 en PubMed-Medline, Scielo y Google, identificó 27 estudios en ALC, que evaluaron la asociación entre dieta y CM. Resultados. La investigación sugiere un efecto protector de ciertos vegetales, frutas, pescado, fibra, vitamina $B_{12}$ y folato, varios fitoestrógenos, licopeno y grasas poliinsaturadas, así como un riesgo potencial por la elevada ingestión calórica, consumo de carnes rojas, procesadas y procesos de cocción, leche y productos lácteos, grasas saturadas y sacarosa. Conclusiones. El impacto del consumo de alimentos y nutrimentos específicos sobre la incidencia del CM no es concluyente, por lo cual se requieren más estudios específicos.

Palabras clave: cáncer de mama; dieta; Latinoamérica
Torres-Sánchez L, Galván-Portillo M, Lewis S,

Gómez-Dantés H, López-Carrillo L.

Diet and breast cancer in Latin-America.

Salud Publica Mex 2009;5 I suppl 2:SI8I-SI 90.

\section{Abstract}

Breast cancer $(B C)$ is the primary cause of death from malignant tumors in women worldwide. Objective. Review epidemiological studies from the Latin-America and Caribbean (LAC) region. Material and Methods. A systematic search, carried out during June-November 2008 in Mexico of PubMed/Medline, Scielo, and Google identified 27 epidemiological studies in LAC that evaluated associations between diet and BC. Results. The research on diet and BC suggests the protective effect of certain fruits, vegetables, fish, fiber, vitamin $B_{12}$, folate, various phytoestrogens, lycopene, and polyunsaturated fats; as well as the potential risk associated with elevated caloric consumption, consumption of red meat and processed meats, certain meat cooking techniques, milk and some other dairy products, saturated fat, and sucrose. Conclusions. The impact of specific foods and nutrients on breast cancer incidence is inconclusive. Further research is needed on this topic.

Key words: Breast cancer; diet; Latin America
L a notoria variabilidad geográfica de la incidencia del cáncer mamario (CM) en el mundo fue una de las primeras observaciones para sugerir que las diferencias en los hábitos dietéticos podían relacionarse con el desarrollo de dicha neoplasia. El efecto de la migración en el riesgo de CM añadió más información en este sentido, ya que las mujeres japonesas residentes en Japón con un bajo riesgo de CM lo incrementaron al emigrar a Hawai, y aún más al nacer y permanecer en Estados Unidos de América, ${ }^{1}$ lo cual sugiere que, entre otros

Este estudio fue posible gracias al apoyo fiinanciero de Instituto Carso de la Salud y el Consejo Promotor Competitividad y Salud de la Fundación Mexicana para la Salud.

(I) Instituto Nacional de Salud Pública, Dirección de Salud Reproductiva. Cuernavaca, Morelos, México.

(2) Fundación Mexicana para la Salud, Observatorio de la Salud. México, D.F.

Fecha de recibido: 31 de octubre de 2008 - Fecha de aprobado: 19 de diciembre de 2008 
factores ambientales, el cambio de la dieta oriental a la occidental es un determinante de dicho riesgo.

Los alimentos contienen diferentes nutrimentos y compuestos que pueden iniciar, acelerar o incluso detener el desarrollo de un tumor maligno. En el último consenso mundial sobre dieta y cáncer se identificaron alimentos y nutrimentos específicos sin lugar a dudas vinculados con el desarrollo de diversos tipos de cáncer, como el gástrico, colónico y rectal, etc. No obstante, la información es todavía incompleta para el $\mathrm{CM}^{2}$ por lo cual deben llevarse a cabo más estudios al respecto.

En Latinoamérica, al igual que en el resto del mundo, el CM ocupa un lugar prioritario como causa de muerte por tumores malignos entre las mujeres. Asimismo, se observan diferencias contrastantes en las tasas de incidencia; basta mencionar que las tasas de Uruguay y Argentina son tres veces mayores que en México ${ }^{3}$ y que los hábitos dietéticos también tienen grandes diferencias. Por ejemplo, el consumo per cápita de carne en los primeros es en promedio de $80 \mathrm{~kg}$ por año, mientras que en México es de $58.4 \mathrm{~kg}$ por año. ${ }^{4}$

El presente documento contiene una revisión de los estudios epidemiológicos más relevantes sobre dieta y CM en Latinoamérica, que podría ser de utilidad en la planeación y desarrollo de futuras investigaciones que se requieren para dilucidar el impacto de la dieta y, en su caso, coadyuvar a prevenir dicha tumoración.

\section{Identificación de los estudios}

Mediante una búsqueda sistemática realizada en México entre junio y noviembre de 2008, en las bases de datos PubMed-Medline, Scielo y Google académico, se identificaron 27 estudios epidemiológicos analíticos ${ }^{5-31}$ provenientes de alguno de los países que conforman la región de América Latina y El Caribe (ALC), publicados hasta el 31 de agosto de 2008, y en los que se evaluó el consumo de alimentos, nutrimentos, compuestos presentes en los alimentos y otros factores de riesgo relacionados con la dieta y su nexo con el cáncer de mama. Como palabras clave en inglés y español se incluyeron cáncer de mama, factor de riesgo, dieta, nutrimentos y cada uno de los países de la región (Argentina, Belice, Bolivia, Brasil, Chile, Colombia, Costa Rica, Cuba, Ecuador, Guatemala, Haití, Honduras, Jamaica, México, Nicaragua, Panamá, Paraguay, Perú, Puerto Rico, República Dominicana, Trinidad y Tobago, Uruguay y Venezuela). Un estudio piloto, sin datos concluyentes, se excluyó por contar con un tamaño de muestra muy pequeño (18 casos y 18 controles). ${ }^{31}$

\section{Metodología de la revisión}

Mediante un formato especialmente diseñado para este fin, dos integrantes del equipo realizaron un registro independiente de las características de cada uno de los estudios. La revisión se dividió en tres secciones: a) estudios que evaluaron la relación entre el consumo de alimentos y el riesgo de cáncer de mama; b) estudios que evaluaron la relación entre el consumo de nutrimentos y el riesgo de cáncer de mama; y c) estudios que evaluaron factores vinculados con la dieta y su relación con el cáncer de mama. El informe de los principales hallazgos se realizó en orden cronológico ascendente, por autor y país de origen, tipo de diseño, tamaño de muestra, razones de momios (RM) notificadas para las categorías de mayor y menor exposición, la condición de menopausia, el significado de la medida de relación y la prueba de tendencia. Asimismo, se describieron para cada estudio las variables consideradas en el análisis o el diseño como potenciales factores de confusión.

Debido a que entre los estudios existen notorias diferencias metodológicas que pueden afectar las relaciones evaluadas, no se calcularon las medidas sumarias de efecto. Entre las principales diferencias observadas se encontraron las siguientes: a) los tipos de alimentos o nutrimentos evaluados, b) los puntos de corte usados en cada uno de ellos (sí o no; terciles, cuartiles; alto o bajo); c) falta de estratificación por estado menopáusico; y d) control por factores de confusión potenciales.

\section{Resultados}

En total se identificaron 27 estudios espidemiológicos sobre dieta y $\mathrm{CM}$. Los países con mayor número de estudios publicados son Uruguay-9,11,12,14,17,18 y México ${ }^{10,13,19-}$ 23,30 (33 y 30\% de las publicaciones, respectivamente) (cuadro I).

En Uruguay, los estudios se han enfocado sobre todo en el consumo de carnes y sus métodos de preparación. Los resultados sugieren que no sólo el consumo de carne sino también su origen (blanca o roja) y la forma de cocinarla (frita, cocida, asada, etc.) son factores que se relacionan en grado significativo con la incidencia de $\mathrm{CM}, 7,8,12$ lo cual se corroboró en un estudio reciente que analizó los tipos de dietas (occidental, tradicional, etc.) y notifica que las mujeres que padecen $\mathrm{CM}$ tienen un patrón de dieta occidentalizado (carne frita, a la parrilla y carnes procesadas), en comparación con las mujeres que no desarrollan $\mathrm{CM} .{ }^{14} \mathrm{El}$ incremento del riesgo de CM vinculado con el consumo de carne roja (figura 1) se 


\section{Cuadro I}

Estudios SOBRE DIETA Y CÁNCER DE MAMA REALIZAdOS EN LATINOAMÉRICA

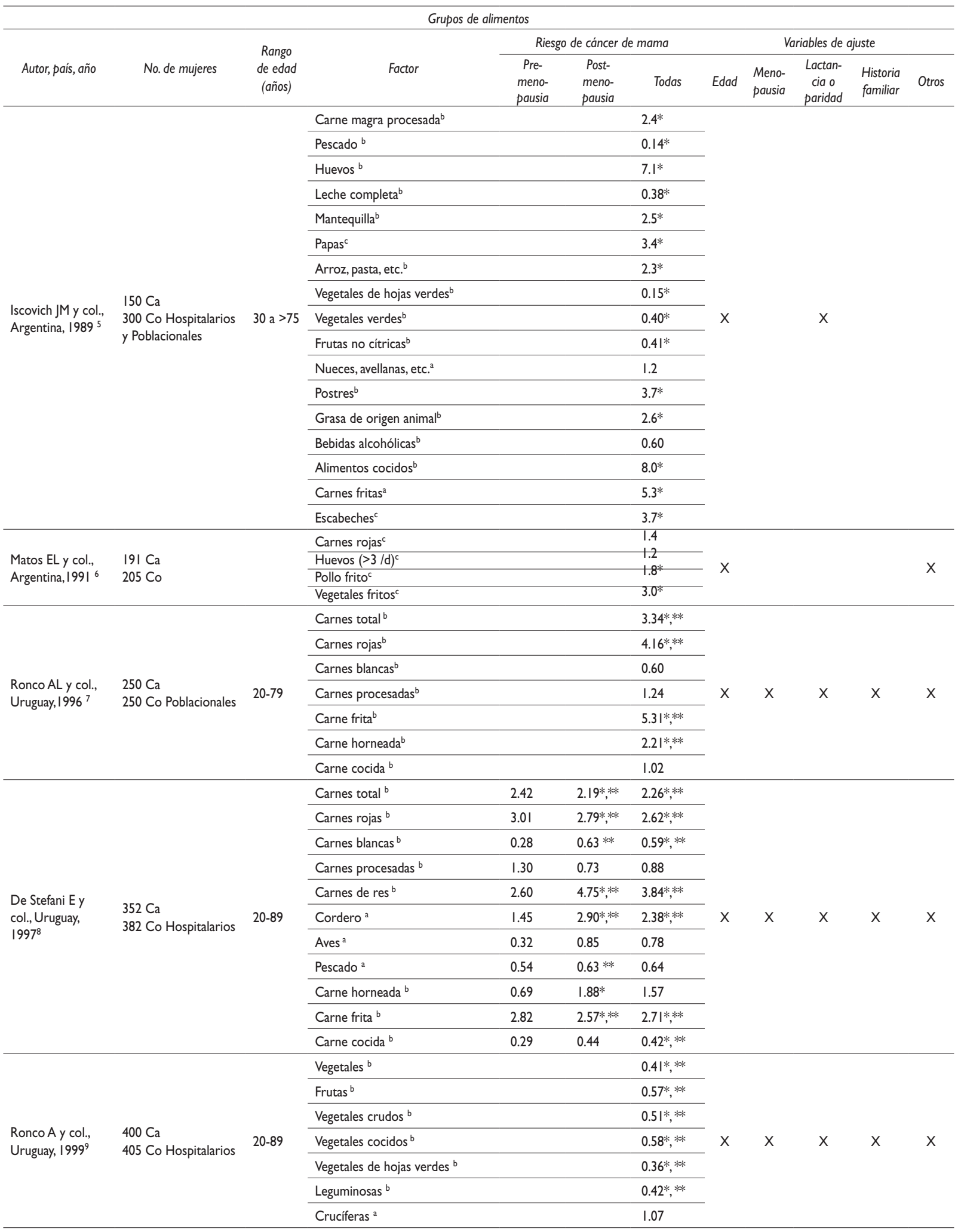


Continuación ...

\begin{tabular}{|c|c|c|c|c|c|c|c|c|c|c|c|}
\hline \multicolumn{12}{|c|}{ Grupos de alimentos } \\
\hline \multirow{3}{*}{ Autor, país, año } & \multirow{3}{*}{ No. de mujeres } & \multirow{3}{*}{$\begin{array}{l}\text { Rango } \\
\text { de edad } \\
\text { (años) }\end{array}$} & \multirow{3}{*}{ Factor } & \multirow{2}{*}{\multicolumn{3}{|c|}{ Riesgo de cáncer de mama }} & \multirow{2}{*}{\multicolumn{5}{|c|}{ Variables de ajuste }} \\
\hline & & & & & & & & & & & \\
\hline & & & & $\begin{array}{l}\text { Pre- } \\
\text { meno- } \\
\text { pausia }\end{array}$ & $\begin{array}{l}\text { Post- } \\
\text { meno- } \\
\text { pausia }\end{array}$ & Todas & Edad & $\begin{array}{l}\text { Meno- } \\
\text { pausia }\end{array}$ & $\begin{array}{l}\text { Lactan- } \\
\text { cia } 0 \\
\text { paridad }\end{array}$ & $\begin{array}{l}\text { Historia } \\
\text { familiar }\end{array}$ & Otros \\
\hline \multirow{11}{*}{$\begin{array}{l}\text { Torres-Sánchez } \\
\text { L y col., México, } \\
2000^{10}\end{array}$} & \multirow{11}{*}{$\begin{array}{l}198 \mathrm{Ca} \\
\text { 198Co Hospitalarios }\end{array}$} & \multirow{11}{*}{$21-79$} & Vegetales de hojas verdes/amarrilla a & & & 0.56 & \multirow{11}{*}{$x$} & \multirow{11}{*}{$x$} & \multirow{11}{*}{$\mathrm{X}$} & \multirow{11}{*}{$\mathrm{X}$} & \multirow{11}{*}{$\mathrm{X}$} \\
\hline & & & Vegetales amarillas/naranjas ${ }^{a}$ & & & 0.79 & & & & & \\
\hline & & & Frutas cítricas ${ }^{a}$ & & & $0.44^{*}, * *$ & & & & & \\
\hline & & & Frutas no cítricas a & & & 0.80 & & & & & \\
\hline & & & $\begin{array}{l}\text { Pescado > } 1.5 \text { porciones/sem vs } \\
\text { nunca }\end{array}$ & & & 0.67 & & & & & \\
\hline & & & $\begin{array}{l}\text { Carnes rojas } \geq 1 \text { porcion/dia vs } \\
<1 / \text { dia }\end{array}$ & & & 1.34 & & & & & \\
\hline & & & Cebolla $\geq$ I rebanada/dia vs $<$ I/dia & $0.18^{*}, * *$ & $0.37^{*}, * *$ & $0.27 *, * *$ & & & & & \\
\hline & & & Lechuga $>$ I hoja/sem vs <l/sem & $0.66 * *$ & 0.73 & $0.70 * *$ & & & & & \\
\hline & & & Espinacas $\geq .5 \mathrm{taza} / \mathrm{sem}$ vs $<.5 / \mathrm{sem}$ & $0.44^{*}, * *$ & 1.39 & 0.85 & & & & & \\
\hline & & & Manzana $\geq 1$ pieza/sem vs $<1 /$ sem & 0.57 & 0.97 & 0.83 & & & & & \\
\hline & & & $\begin{array}{l}\text { Té de hierbas } \geq 3 \text { tazas/sem vs }<3 \text { / } \\
\text { sem }\end{array}$ & 0.68 & 1.54 & 1.04 & & & & & \\
\hline \multirow{13}{*}{$\begin{array}{l}\text { Ronco AL y col., } \\
\text { Uruguay, } 2002^{\prime \prime}\end{array}$} & \multirow{13}{*}{$\begin{array}{l}\text { III Ca } \\
222 \mathrm{Co} \text { Hospitalarios }\end{array}$} & \multirow{13}{*}{$\begin{array}{l}\leq 49 \\
\mathrm{a} \\
\geq 70\end{array}$} & Leche completa ${ }^{a}$ & & & $2.84 * * *$ & \multirow{13}{*}{$\mathrm{X}$} & \multirow{13}{*}{$\mathrm{x}$} & \multirow{13}{*}{$\mathrm{x}$} & \multirow{13}{*}{$\mathrm{X}$} & \\
\hline & & & Leche descremada a & & & 0.89 & & & & & \\
\hline & & & Leche con chocolate $^{\text {a }}$ & & & $2.85 * * *$ & & & & & \\
\hline & & & Yogurt entero $^{a}$ & & & 1.03 & & & & & \\
\hline & & & Yogurt descremado a & & & $0.29 *$, ** & & & & & \\
\hline & & & Ricota ${ }^{a}$ & & & $0.45^{*}$ & & & & & \\
\hline & & & Mozarela $^{a}$ & & & 1.12 & & & & & \\
\hline & & & Dambo a & & & 1.66 & & & & & \\
\hline & & & Parmesano $^{a}$ & & & 1.17 & & & & & \\
\hline & & & Gruyere $^{\mathrm{a}}$ & & & 1.93*** & & & & & \\
\hline & & & Mantequilla ${ }^{a}$ & & & 0.92 & & & & & \\
\hline & & & Helado a & & & $1.98 * * *$ & & & & & \\
\hline & & & Crema chantilly a & & & 1.18 & & & & & \\
\hline & & & Pollo con piel ${ }^{\mathrm{c}}$ & & & 1.54 & & & & & \\
\hline & & & Pollo sin piel ${ }^{\mathrm{c}}$ & & & $0.42 *$ & & & & & \\
\hline Ronco AL y col., & III Ca & $\leq 49$ & Pescado frito ${ }^{c}$ & & & $1.99 *$ & $x$ & & $x$ & $x$ & $x$ \\
\hline Uruguay, $2003^{12}$ & 222 Co Hospitalarios & $\geq 70$ & Pescado no frito ${ }^{c}$ & & & $0.48 *$ & $\wedge$ & & 入 & 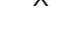 & ^ \\
\hline & & & Carne blanca magra $^{c}$ & & & $0.34 *$ & & & & & \\
\hline & & & Carne blanca grasosa ${ }^{c}$ & & & 1.62 & & & & & \\
\hline Lajous M y col., & $475 \mathrm{Ca}$ & 1897 & Carga glicémica ${ }^{\mathrm{b}}$ & 1.43 & $2.18^{*}, * *$ & 1.62*,** & $x$ & $x$ & $\mathrm{x}$ & $x$ & $x$ \\
\hline México, $2005^{13}$ & nales & $10-01$ & Indice glicémico b & 0.66 & 0.81 & 0.84 & $\lambda$ & $\lambda$ & $\lambda$ & $\lambda$ & $\lambda$ \\
\hline & & & Dieta tradicional ${ }^{c}$ & 0.80 & $0.73^{*}$ & $0.53 *$, $* *$ & & & & & \\
\hline & & & Saludable ${ }^{c}$ & 1.49 & $0.72^{*}$ & $0.46 *$, ** & & & & & \\
\hline Ronco AL y col., & $442 \mathrm{Ca}$ & 3080 & Occidental $^{\mathrm{c}}$ & 1.17 & 1.38 & $2.16 * * *$ & & & & & \\
\hline Uruguay, $2006^{14}$ & 442 Co Hospitalarios & 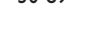 & Cocido $^{c}$ & 0.88 & $0.8 I^{*}$ & 0.71 & . & 人 & 人 & 人 & \\
\hline & & & Alta en grasa ${ }^{c}$ & 0.84 & 1.16 & 1.38 & & & & & \\
\hline & & & Alcohol $^{\mathrm{c}}$ & 1.03 & 1.04 & 1.06 & & & & & \\
\hline
\end{tabular}


Continuación ...

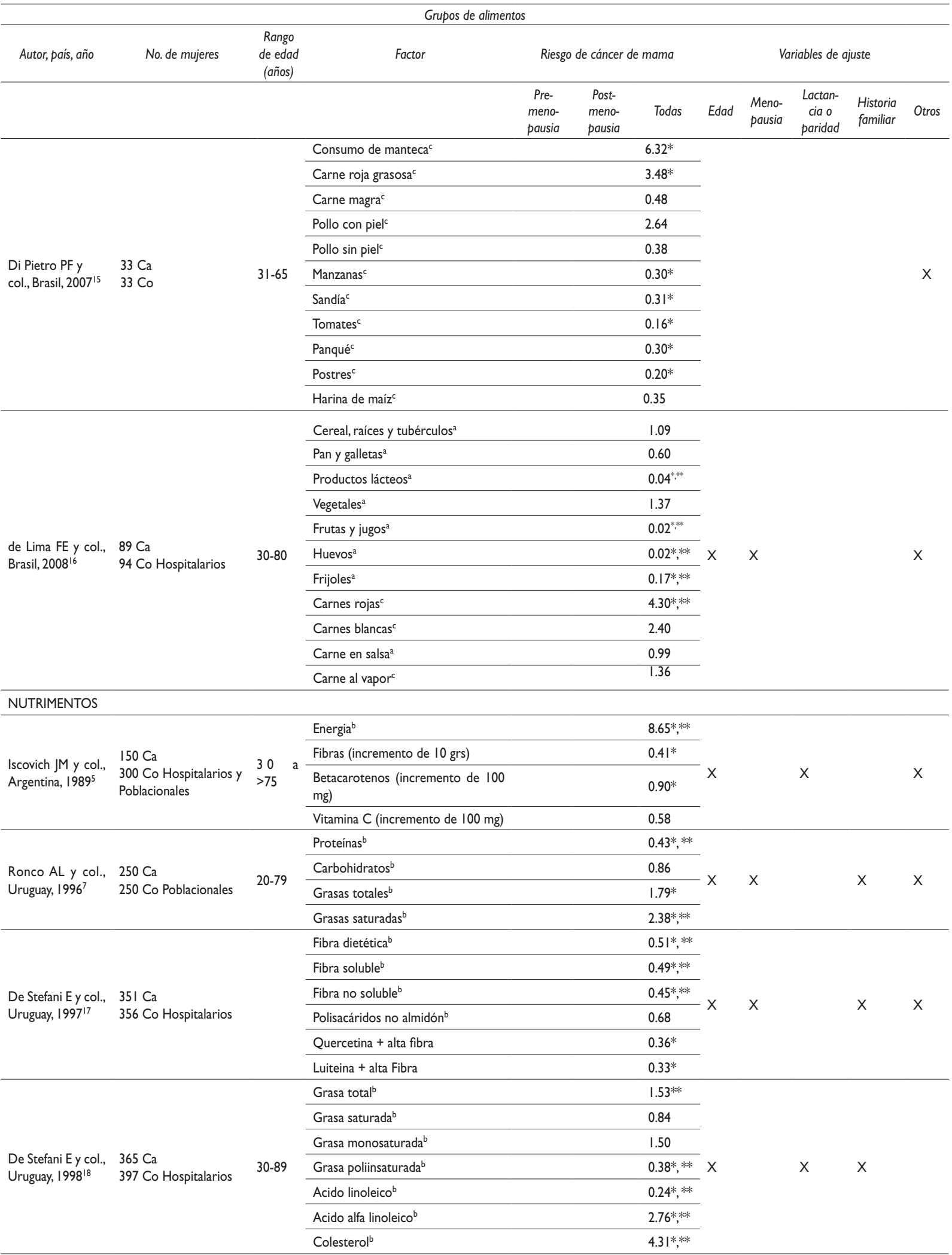


Continuación ...

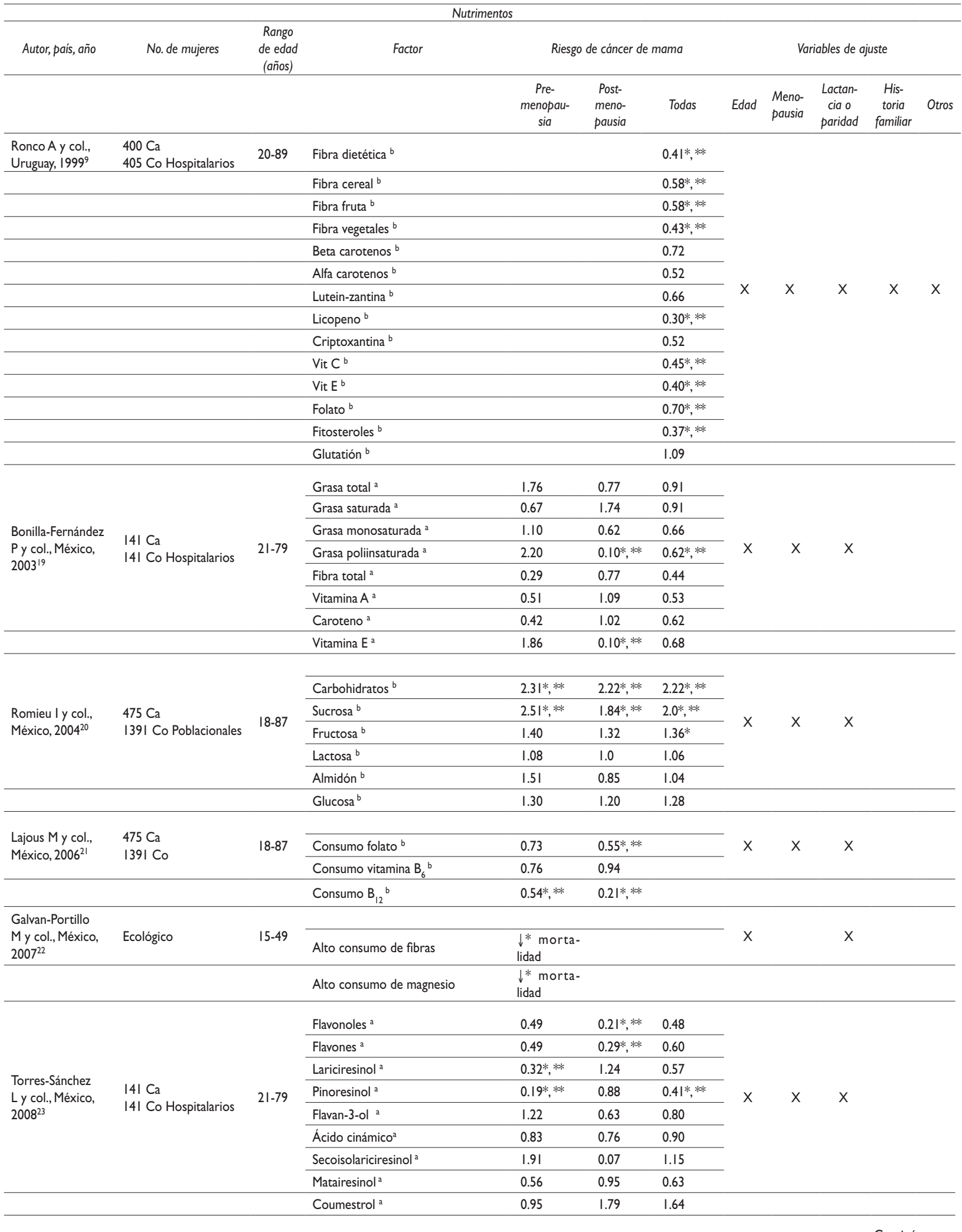


Continuación ...

\begin{tabular}{|c|c|c|c|c|c|c|c|c|c|c|c|}
\hline \multicolumn{12}{|c|}{ Otros factores vinculados con la dieta } \\
\hline \multirow{2}{*}{ Autor, país, año } & \multirow{2}{*}{ No. de mujeres } & \multirow{2}{*}{$\begin{array}{c}\text { Rango } \\
\text { de edad } \\
\text { (años) }\end{array}$} & \multirow{2}{*}{ Factor } & \multicolumn{3}{|c|}{ Riesgo de cáncer de mama } & \multicolumn{5}{|c|}{ Variables de ajuste } \\
\hline & & & & $\begin{array}{c}\text { Pre- } \\
\text { meno- } \\
\text { pausia }\end{array}$ & $\begin{array}{l}\text { Post- } \\
\text { meno- } \\
\text { pausia }\end{array}$ & Todas & Edad & $\begin{array}{l}\text { Meno- } \\
\text { pausia }\end{array}$ & $\begin{array}{l}\text { Lactan- } \\
\text { cia o } \\
\text { paridad }\end{array}$ & $\begin{array}{l}\text { Historia } \\
\text { familiar }\end{array}$ & Otros \\
\hline \multirow{3}{*}{$\begin{array}{l}\text { De Stefani E y col., } \\
\text { Uruguay, } 1997^{8}\end{array}$} & \multirow{3}{*}{$\begin{array}{l}352 \mathrm{Ca} \\
382 \text { Co Hospitalarios }\end{array}$} & \multirow{3}{*}{$20-89$} & Amina heterocíclica $\mathrm{IQ}^{\mathrm{b}}$ & 2.15 & $3.80 * * *$ & \multirow{3}{*}{$\begin{array}{l}3.34^{*}, * * \\
2.13^{*}, * * \\
2.59 * * *\end{array}$} & \multirow{3}{*}{$x$} & \multirow{3}{*}{$x$} & \multirow{3}{*}{$x$} & \multirow{3}{*}{$x$} & \multirow{3}{*}{$x$} \\
\hline & & & Amina heterocíclica MelQx ${ }^{b}$ & 1.97 & 2.14*** & & & & & & \\
\hline & & & Amina heterocíclica $\mathrm{PHiP}{ }^{b}$ & 1.16 & $3.31 * * *$ & & & & & & \\
\hline \multirow{11}{*}{$\begin{array}{l}\text { Schreier L y col., } \\
\text { Chile, } 1999^{24}\end{array}$} & \multirow{11}{*}{$\begin{array}{l}30 \mathrm{Ca} \\
30 \mathrm{Co}\end{array}$} & \multirow{11}{*}{$28-72$} & Triglicéridos $(\mathrm{mg} / \mathrm{dl})^{\mathrm{e}}$ & III vs. 7I* & $\begin{array}{c}141 \\
\text { vs. } 105 *\end{array}$ & $\begin{array}{c}127 \\
\text { vs. } 88^{*}\end{array}$ & \multirow{11}{*}{$x$} & \multirow{11}{*}{$x$} & & & \\
\hline & & & Colesterol $(\mathrm{mg} / \mathrm{dl})^{\mathrm{e}}$ & 197 vs. 214 & $\begin{array}{c}232 \\
\text { vs. } 259\end{array}$ & $\begin{array}{c}217 \\
v s .236\end{array}$ & & & & & \\
\hline & & & Colesterol LDL $(\mathrm{mg} / \mathrm{dl})^{\mathrm{e}}$ & 119 vs. 125 & $\begin{array}{c}153 \\
\text { vs. } 165\end{array}$ & $\begin{array}{c}138 \\
\text { vs. } 145\end{array}$ & & & & & \\
\hline & & & Colesterol HDL total $(\mathrm{mg} / \mathrm{dl})^{\mathrm{e}}$ & 57 vs. $72 *$ & 57 vs. $72^{*}$ & $\begin{array}{c}56 \\
\text { vs. } 73 *\end{array}$ & & & & & \\
\hline & & & Colesterol HDL ${ }_{2}(\mathrm{mg} / \mathrm{dl})^{\mathrm{e}}$ & $\begin{array}{c}12.3 \\
\text { vs. } 19.8 *\end{array}$ & $\begin{array}{l}7.0 \\
\text { vs. } 13.5^{*}\end{array}$ & $\begin{array}{c}9.9 \\
\text { vs. } 16.7^{*}\end{array}$ & & & & & \\
\hline & & & Colesterol HDL $\mathrm{H}_{3}(\mathrm{mg} / \mathrm{dl})^{\mathrm{e}}$ & $\begin{array}{c}44.7 \\
\text { vs. } 52.2\end{array}$ & $\begin{array}{c}48.0 \\
\text { vs } 59.5^{*}\end{array}$ & $\begin{array}{c}46.1 \\
\text { vs.56.3* }\end{array}$ & & & & & \\
\hline & & & Apo Lipoproteínas A (mg/dl) e & I 47 vs. 154 & $\begin{array}{c}157 \\
\text { vs. } 145\end{array}$ & $\begin{array}{c}153 \\
\text { vs. } 149\end{array}$ & & & & & \\
\hline & & & Apo Lipoproteínas B (mg/dl) e & 120 vs. 117 & $\begin{array}{c}147 \\
\text { vs. } 133\end{array}$ & $\begin{array}{c}134 \\
\text { vs. } 125\end{array}$ & & & & & \\
\hline & & & Índice de masa corporal ${ }^{\mathrm{e}}$ & 24 vs. 21.5 & 29 vs. 25 & & & & & & \\
\hline & & & Cintura/cadera ${ }^{\mathrm{e}}$ & $\begin{array}{l}0.8 \text { vs. } \\
0.7 I^{*}\end{array}$ & $\begin{array}{c}0.85 \\
\text { vs.0.78* }\end{array}$ & & & & & & \\
\hline & & & Insulina en ayunas ${ }^{e}$ & & & $\begin{array}{r}15.9 \\
\text { vs.8.9* }\end{array}$ & & & & & \\
\hline \multirow{2}{*}{$\begin{array}{l}\text { Mendonça GA y } \\
\text { col., Brasil, } 1999^{25}\end{array}$} & \multirow{2}{*}{$\begin{array}{l}177 \mathrm{Ca} \\
350 \mathrm{Co}\end{array}$} & \multirow{2}{*}{$30-75$} & Indice de masa corporal ${ }^{\text {a }}$ & & & 0.54 & \multirow{2}{*}{$x$} & & $\mathrm{x}$ & $\mathrm{x}$ & $\mathrm{x}$ \\
\hline & & & Alcohol $^{b}$ & & & $2.15^{*}$ & & & $\lambda$ & $\lambda$ & $\lambda$ \\
\hline & & & Indice de masa corporal $\geq 30$ & & & $1.79 *$ & & & & & \\
\hline $\begin{array}{l}\text { Atalah E y col., } \\
\text { Chile, } 2000^{26}\end{array}$ & $\begin{array}{l}170 \mathrm{Ca} \\
340 \mathrm{Co}\end{array}$ & $>20$ & Consumo de alcohold & & & $1.61^{*}$ & $x$ & & & & \\
\hline & & & Tabaquismo & & & 1.10 & & & & & \\
\hline & & & Talla $\geq 160$ vs. $<152 \mathrm{~cm}^{b}$ & 154 & 1.29 & & & & & & \\
\hline & & & IMC actual $\geq 30.2^{\mathrm{b}}$ & $0.25 * * *$ & 0.61 & & & & & & \\
\hline & & & IMC 18 años ${ }^{b}$ & 0.50 & 0.84 & & & & & & \\
\hline & & & IMC 30 años $^{b}$ & 0.31 & 0.82 & & & & & & \\
\hline $\begin{array}{l}\text { de Vasconcelos } \\
\text { AB y col., Brasil, }\end{array}$ & $177 \mathrm{Ca}$ & $<75$ & IMC máximob & $0.15 * * *$ & 0.92 & & $x$ & $x$ & $x$ & $\mathrm{X}$ & $x$ \\
\hline $2001^{27}$ & 377 Co & & $\begin{array}{l}\text { Pérdida de peso desde los } 18 \\
\text { años }{ }^{d}\end{array}$ & $16.65^{*}, * *$ & 2.05 & & & & & & \\
\hline & & & $\begin{array}{l}\text { Pérdida de peso de los 18-30 } \\
\text { años }{ }^{d}\end{array}$ & $29.02 *$ & 1.57 & & & & & & \\
\hline & & & $\begin{array}{l}\text { Pérdida de peso desde los } 30 \\
\text { años }{ }^{d}\end{array}$ & 0.72 & 0.98 & & & & & & \\
\hline Garmendia ML y & $170 \mathrm{CaMa}$ & & $\begin{array}{l}\text { Resistencia a la insulina HOMA } \\
>2.5\end{array}$ & 0.84 & $2.70^{*}$, , *k & 1.4 & & & & & \\
\hline col., Chile, $2007^{28}$ & y & $33-86$ & Insulina $(\mathrm{mg} / \mathrm{dL})$ & 1.06 & 1.01 & 1.02 & $x$ & & & & $x$ \\
\hline & & & Glucosa (mg/dL) & 1.03 & $1.01 * * * *$ & $1.01 *$ & & & & & \\
\hline $\begin{array}{l}\text { Lobelo Fy col., } \\
\text { Colombia, } 2006^{29}\end{array}$ & Ecológico & $>45 \mathrm{a}$ & Act. física & & & $0.76^{*}$ & $x$ & & & & \\
\hline Ortíz-Rodríguez & $58 \mathrm{Ca}$ & & Act fisica Moderada (hrs/sem) & 0.99 & $0.91 *$, * & & & & & & \\
\hline $\begin{array}{l}\text { S y cols., México, } \\
2008^{30}\end{array}$ & $\begin{array}{l}58 \text { Co Poblacionales y } \\
\text { Hospitalarios }\end{array}$ & & $\begin{array}{l}\text { Act. fisica total (METs/hora- } \\
\text { semana) }\end{array}$ & 0.95 & $0.88^{*},{ }^{*}$ & & $x$ & $x$ & $x$ & & $x$ \\
\hline
\end{tabular}

\footnotetext{
* $\quad p<0.05$

$\mathrm{c}=$ Alto vs. bajo

* $p$ de la prueba de tendencia $<0.05$

$d=$ Sí vs. no

$=T_{3}$ vs.T

$\mathrm{b}=\mathrm{Q}_{4}$ vs. $\mathrm{Q}_{\text {। }}$

$\mathrm{e}=\mathrm{T}_{3} v s, \mathrm{~T}_{1}$

$\mathrm{f}=\mathrm{Q}_{4} \mathrm{vs} \cdot \mathrm{Q}_{\mathrm{I}}$
} 


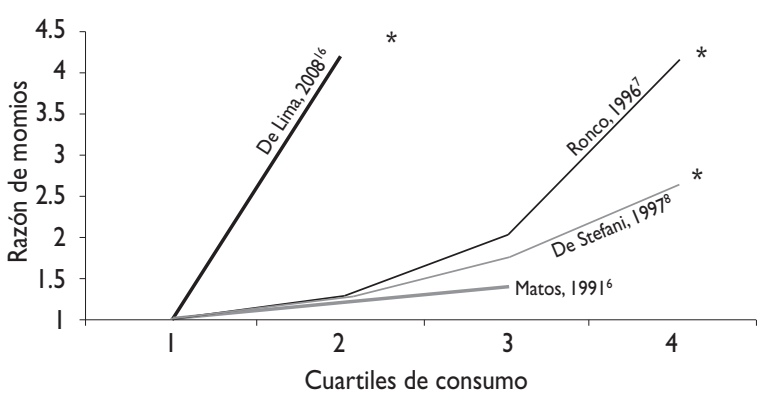

$\begin{array}{cr}\text { De Stefani, } 1997^{8} & \text { Ronco, } 1996^{7} \\ \text { De Lima, 2008 } & \text { Matos, } 1991^{6}\end{array}$

* " $p$ " de la prueba de tendencia $<0.05$

Figura I. Riesgo de cáncer de mama asociado al CONSUMO de CARNes Rojas en países de AmÉrica Latina y EL Caribe

ha notificado de forma consistente también en Brasi ${ }^{16}$ y Argentina. ${ }^{6} \mathrm{Al}$ respecto, en uno de los estudios en el que se estratificó por estado menopáusico, se identificó un incremento del riesgo significativo entre las posmenopáusicas respecto de las premenopáusicas. ${ }^{8}$

Los mecanismos subyacentes propuestos para la relación entre carnes rojas y CM se basan en la producción de compuestos nitrosos y la liberación de hierro. Los compuestos nitrosos se generan durante la digestión como consecuencia de las bacterias presentes en el estómago y el intestino grueso, así como durante el proceso de cocción, en el cual las elevadas temperaturas favorecen la generación de aminas heterocíclicas e hidrocarburos aromáticos policíclicos. De manera adicional, durante el proceso de cocción el grupo hem libera hierro, que favorece la producción de radicales libres, activa los factores de transcripción encargados de la respuesta oxidativa, las citocinas proinflamatorias y las señales de hipoxia. ${ }^{2}$

En contraste, el consumo de carotenos, vitaminas $\mathrm{C}$ y E, entre otras, se ha vinculado con un decremento del riesgo de CM en Uruguay, ${ }^{9}$ que es consistente con el efecto protector de la ingestión de vegetales de hojas verdes y frutas no cítricas, identificado en Argentina, y el consumo de manzana y sandía en Brasil. ${ }^{15}$

En México se ha reconocido que el consumo de cebolla y espinaca, ${ }_{10}^{10}$ grasas poliinsaturadas y vitamina $\mathrm{E}_{,}{ }^{19}$ vitamina $\mathrm{B}_{12}$ y folato, ${ }^{21}$ además de flavonoles, flavones y otros fitoestrógenos, ${ }^{23}$ reduce de modo considerable el riesgo de $\mathrm{CM}$; en contraste, el consumo de hidratos de carbono y en particular la sacarosa lo incrementa. ${ }^{20} \mathrm{En}$ relación con la mortalidad, un estudio ecológico reciente sugiere que el alto consumo de fibras y magnesio ${ }^{22}$ reduce en grado sustancial la mortalidad por CM.

La cebolla, al igual que la espinaca y muchos otros alimentos de origen vegetal, son ricos en fitoquímicos, los cuales ejercen su función protectora a través de varios mecanismos: antioxidantes, antiproliferativos, promotores de la apoptosis, y también como antiestrógenos, debido a su afinidad por los receptores estrogénicos, sobre todo el receptor estrogénico de tipo beta. ${ }^{32}$ Por su parte, el papel protector del folato ${ }^{33} \mathrm{y}$ otros micronutrimentos (p. ej., magnesio) ${ }^{34}$ se debe a su participación en la síntesis, estabilidad y reparación del ADN, mecanismos cuya alteración se relaciona con el proceso de carcinogénesis.

En cuanto al consumo de alcohol, en el plano mundial se considera que la evidencia disponible es convincente en relación con el incremento del riesgo de $\mathrm{CM}$, tanto en mujeres premenopáusicas como posmenopáusicas, y se calcula que existe un incremento del riesgo de alrededor de $10 \%$ por el consumo de $10 \mathrm{mg}$ por día de etanol..$^{35}$ En la región de ALC sólo dos estudios, uno en Brasil ( $O R=2.15 ;$ IC95\% 1.04-4.25 > 10 copas / año $)^{25}$ y otro en Chile $(O R=1.61$; IC95\% 1.06-2.54, consumo excesivo, sí o no $)^{26}$, señalan un aumento del riesgo de $\mathrm{CM}$ vinculado con el consumo de alcohol.

Las bebidas alcohólicas pueden contener sustancias carcinógenas (acetaldehídos), además de que el alcohol por sí mismo puede solubilizar otros carcinógenos externos para que penetren en la célula. Asimismo, se ha demostrado que el alcohol modifica el metabolismo y la acción de los estrógenos y sus efectos pueden tener la mediación de la generación de radicales libres. ${ }^{2}$

\section{Factores relacionados con la dieta y el riesgo de cáncer de mama}

La obesidad es el resultado de un desequilibrio entre la ingestión y el gasto calórico, este último determinado en especial por la actividad física. El mecanismo propuesto es un incremento de la reacción inflamatoria corporal, y como consecuencia de los niveles circulantes de hormonas, como insulina, factores de crecimiento semejantes a la insulina (IGF) y estrógenos. Esto crea un ambiente que promueve la carcinogénesis e inhibe la apoptosis. ${ }^{36}$

El CM se ha estudiado en relación con la obesidad, la actividad física y, en fecha más reciente, con patrones metabólicos vinculados con la ingestión de nutrimentos que aportan calorías, como los hidratos de carbono. Aunque los mecanismos no se han definido con claridad, se ha identificado que la obesidad, evaluada a través del índice de masa corporal (IMC), es un factor de riesgo para el CM en las mujeres posmenopáusicas y un factor 
protector en las mujeres premenopáusicas. ${ }^{2}$ En la región de ALC, uno de los estudios que evaluó el IMC reporta en todas las mujeres un riesgo de $\mathrm{CM}$ cerca de dos veces mayor en mujeres con un IMC $\geq 30 .{ }^{26}$ En contraste, en Brasil $^{27}$ se observó una disminución significativa del riesgo de $\mathrm{CM}$ con un $\mathrm{IMC}$ actual y máximo $\geq 30$ en mujeres premenopáusicas. En Argentina, la obesidad evaluada por medio del índice cintura-cadera se relacionó con un riesgo significativamente mayor de $\mathrm{CM}$ en mujeres premenopáusicas y posmenopáusicas. ${ }^{24}$

Otros factores que son determinantes de la obesidad incluyen la ingestión de alimentos con alto índice glucémico, la hiperglucemia y la resistencia a la insulina. La ingestión de hidratos de carbono incrementa la respuesta glucémica e insulínica y, en consecuencia, mediante las IGF altera la proliferación celular y la apoptosis. ${ }^{36}$ En México, la ingestión de una gran carga glucémica se relacionó con un incremento significativo del riesgo de $\mathrm{CM}$ en mujeres posmenopáusicas. ${ }^{13}$ Por su parte, en Chile se ha informado un aumento significativo del riesgo $\mathrm{CM}$ en mujeres posmenopáusicas vinculado con la resistencia a la insulina y la hiperglucemia, ${ }^{28}$ así como una concentración promedio de triglicéridos séricos significativamente mayor entre los casos de CM en comparación con los controles. ${ }^{24}$

Por su parte, la actividad física se ha relacionado con una disminución del riesgo de CM en mujeres posmenopáusicas. No obstante, aunque existen posibles mecanismos biológicos que explicarían dicho efecto (reducción de grasa corporal, metabolismo de hormonas esteroideas exógenas, eficiencia del sistema inmunitario, etc.), la información disponible no permite extrapolarlo a mujeres premenopáusicas. ${ }^{2}$ Un estudio ecológico realizado en Bogotá estimó que 14.2\% de las muertes por CMen mujeres mayores de 45 años se debe a inactividad física. ${ }^{29}$ En México, otro estudio señaló ${ }^{30}$ una reducción del riesgo de $\mathrm{CM}$ por cada hora adicional a la semana de actividad física moderada.

\section{Discusión}

La investigación sobre dieta y CM conducida en diferentes países de Latinoamérica ha contribuido al estado del arte y muestra en particular el efecto protector de los vegetales y frutas, ${ }^{5,9}$ el pescado, ${ }^{12}$ la fibra, ${ }_{1}^{17}$ la vitamina $\mathrm{B}_{12} \mathrm{y}$ el folato, ${ }^{21}$ varios fitoestrógenos (en particular el flaván 3-ol y el pinoresinol), ${ }^{23}$ el licopeno ${ }^{9}$ y las grasas poliinsaturadas, ${ }^{18,19}$ además del incremento del riesgo potencial de la elevada ingestión calórica, ${ }^{5}$ el consumo de carnes rojas, ${ }^{7,8,15,16}$ carnes procesadas, ${ }^{14}$ la forma de preparación de las carnes, ${ }^{12}$ leche y algunos productos lácteos, ${ }^{11}$ grasas saturadas ${ }^{7}$ y sacarosa, ${ }^{20}$ además de compuestos secundarios como las aminas heterocíclicas. ${ }^{8}$
No obstante, es importante mencionar que pese al gran número de proyectos realizados, un gran porcentaje de ellos carece de las características metodológicas que sustente con rigor científico los resultados. De manera específica, la mayor parte incluye sólo estimadores de asociación (razones de momios) y carece de pruebas de tendencia dosis-respuesta y control por variables de confusión importantes, como la lactancia, lo cual limita el alcance de la información que comunican.

Como ya se mencionó, el consenso mundial más reciente considera que la evidencia científica sobre el consumo de alimentos y nutrimentos específicos y su efecto en la incidencia del CM no es concluyente, por lo cual se requieren muchos más estudios en este sentido. Con la finalidad de hacer más efectivos en términos del costo las futuras investigaciones, es posible que se requiera un esfuerzo latinoamericano para diseñar y desarrollar de modo conjunto nuevos y más ambiciosos estudios que evalúen las interacciones de la dieta, con contaminantes y polimorfismos genéticos específicos, que podrían realizarse con base en la experiencia ya existente en la región.

En contraste con otros cánceres, para los cuales es posible la prevención primaria a través de vacunas (cáncer cervicouterino, hepático) o eliminación del tabaco (cáncer de pulmón), la prevención primaria del CMes aún poco factible, en parte debido al conocimiento parcial de los determinantes modificables (esto es, dieta y ejercicio) que lo producen y en su caso el gran reto poblacional que conlleva el cambio de los hábitos dietéticos y la actividad física. Por lo anterior, es evidente la necesidad de incrementar el conocimiento acerca de los factores de riesgo de dicho tumor y fortalecer la prevención secundaria, es decir, el diagnóstico temprano (mamografía), que por el momento es la alternativa factible.

\section{Referencias}

I. Nelson N. Migrant studies aid the search for factors linked to breast cancer risk. J Nat Cancer Inst 2006;98:436-38.

2. World Cancer Research Fund/American Institute for Cancer Research. Food, nutrition, physical activity, and the prevention of cancer: a global perspective. Washington DC:AICR, 2007.

3. Globocan 2002: Data Sources and Methods [database on the Internet]. International Agency for Research on Cancer. 2005 [consultado el I de mayo, 2008]. Disponible en: http://www-dep.iarc.fr.

4. Food and Agriculture Organization of the United Nations (FAO), FAOSTAT on-line statistical service. Food Security Statistics. Disponible en: http://www.fao.org/faostat/foodsecurity/index_es.htm. Consultado el 26 de octubre, 2008.

5. Iscovich JM, Iscovich RB, Howe G, Shiboski S, Kaldor JM.A case-control study of diet and breast cancer in Argentina. Int J Cancer 1989;44:770-776. 6. Matos EL, Thomas DB, Sobel N,Vuoto D. Breast cancer in Argentina: case-control study with special reference to meat eating habits. Neoplasma 1991;38:357-366. 
7. Ronco A, De Stefani E, Mendilaharsu M, Deneo-Pellegrini H. Meat, fat and risk of breast cancer: a case-control study from Uruguay. Int J Cancer 1996;65:328-331.

8. De Stefani E, Ronco A, Mendilaharsu M, Guidobono M, Deneo-Pellegrini H. Meat intake, heterocyclic amines, and risk of breast cancer: a casecontrol study in Uruguay. Can Epidemiol Biomar Prev 1997;6:573-58I. 9. Ronco A, De Stefani E, Boffetta P, Deneo-Pellegrini H, Mendilaharsu $M$, Leborgne F.Vegetables, fruits, and related nutrients and risk of breast cancer: a case-control study in Uruguay. Nutr Cancer 1999;35: I I I I 9. 10. Torres-Sanchez L, Lopez-Carrillo L, Lopez-Cervantes M, Rueda-Neria C,Wolff MS. Food sources of phytoestrogens and breast cancer risk in Mexican women. Nutr Cancer 2000;37:134-139.

II. Ronco AL, De Stefani E, Dáttoli R. Dairy foods and risk of breast cancer: a case-control study in Montevideo, Uruguay. Eur J Cancer Prev 2002; I I:457-463.

12. Ronco AL, De Stefani E, Fabra A. White meat intake and the risk of breast cancer: a case-control study in Montevideo, Uruguay. Nutr Res 2003:23:151-162.

13. Lajous M,Willett W, Lazcano-Ponce E, Sanchez-Zamorano LM, Hernandez-Avila M, Romieu I. Glycemic load, glycemic index, and the risk of breast cancer among Mexican women. Can Caus Control 2005; 16:1165-1169.

14. Ronco AL, De Stefani E, Boffetta P, Deneo-Pellegrini H,Acosta G, Mendilaharsu M. Food patterns and risk of breast cancer:A factor analysis study in Uruguay. Int J Cancer 2006; 1 19:1672-1678.

I5. Di Pietro PF, Medeiros NI,Vieira FG, Fausto MA, Bello-Klein A. Breast cancer in southern Brazil: association with past dietary intake. Nutr Hosp 2007;22:565-572

16. Lima FE, Rosario Dias S, Oliveira Latorre M, Carvalho Costa MJ, Fisberg RM. Diet and cancer in Northeast Brazil: evaluation of eating habits and food group consumption in relation to breast cancer. $\mathrm{Cad}$ Saude Publica 2008;24:820-828.

17. De Stefani E, Correa P, Ronco A, Mendilaharsu M, Guidobono M, Deneo-Pellegrini H. Dietary fiber and risk of breast cancer: a case-control study in Uruguay. Nutr Cancer 1997;28:14-19.

18. De Stefani E, Deneo-Pellegrini H, Mendilaharsu M, Ronco A. Essential fatty acids and breast cancer: a case-control study in Uruguay. Int J Cancer 1998;76:49|-494.

19. Bonilla-Fernandez P, Lopez-Cervantes M, Torres-Sanchez LE, TortoleroLuna G, Lopez-Carrillo L. Nutritional factors and breast cancer in Mexico. Nutr Cancer 2003;45: I 48-I55.

20. Romieu I, Lazcano-Ponce E, Sanchez-Zamorano LM, WillettW, Hernandez-Avila M. Carbohydrates and the risk of breast cancer among Mexican women. Cancer Epidemiol Biomar Prev 2004;13:1283-1289. 21. Lajous M, Lazcano-Ponce E, Hernandez-Avila M,Willett W, Romieu I. Folate, vitamin $B(6)$, and vitamin $B(12)$ intake and the risk of breast cancer among Mexican women. Can Epidemiol Biomar Prev 2006;15:443-448.
22. Galván-Portillo M, Flores A,Torres-Sánchez L, Hernández R, López-Carrillo L. Consumo de micronutrimentos y mortalidad por cáncer mamario en mujeres premenopausicas mexicanas. Cancerología 2007:2:345-350.

23.Torres-Sanchez L, Galvan-Portillo M,Wolff MS, Lopez-Carrillo L. Dietary consumption of phytochemicals and breast cancer risk in Mexican women. Public Health Nutr 2008 Jul 23: I-7. [Epub ahead of print] 24. Schreier LE, Berg GA, Basilio FM, Lopez GI, Etkin AE,Wikinski RL. Lipoprotein alterations, abdominal fat distribution and breast cancer. Biochem Mol Biol Int 1999;47:68I-690.

25. Mendonça GA, Eluf-Neto J,Andrada-Serpa MJ, Carmo PA, Barreto $\mathrm{HH}$, Inomata ON,et al. Organochlorines and breast cancer: a case-control study in Brazil. Int J Cancer 1999;83:596-600.

26. Atalah E, Urteaga C, Rebolledo A, Medina E, Csendes A. Breast cancer risk factors in women fron Santiago, Chile. Rev Med Chile 2000; I 28: I- I I. 27. Vasconcelos AB,Azevedo e Silva Mendonça G, Sichieri R. Height, weight, weight change and risk of breast cancer in Rio de Janeiro,Brazil. Sao Paulo Med J 200 I; I 19:62-66.

28. Garmendia ML, Pereira A,Alvarado ME,Atalah E. Relation between insulin resistance and breast cancer among Chilean women. Ann Epidemiol 2007; 17:403-409.

29. Lobelo F, Pate R, Parra D, Duperly ], Pratt M. [Burden of mortality associated to physical inactivity in Bogota, Colombia]. Rev Salud Publica (Bogota) 2006;8(Suppl 2):28-4I.

30. Ortiz-Rodriguez SP,Torres-Mejia G, Mainero-Ratchelous F,AngelesLlerenas A, Lopez-Caudana AE, Lazcano-Ponce E, et al. [Physical activity and breast cancer risk in Mexican women] Salud Publica Mex 2008;50:126-135.

3I. Santiago E, Gonzalez MJ, Matos MI, Perez CM.Association between dietary fat and breast cancer in Puerto Rican postmenopausal women attending a breast cancer clinic. P R Health Sci J 1998; 17:235-241.

32. Duffy C, Perez K, Partridge A. Implications of phytoestrogen intake for breast cancer. CA Cancer J Clin 2007;57:260-277.

33. Szyfa M, Pakneshanb P, Rabbanib SA. DNA methylation and breast cancer. Biochem Pharmacol 2004;68: I | 87-I I 97.

34. Hartwig A. Role of magnesium in genomic stability. Mutat Res 200I;475:I |3-I2|

35. Hamajima N, Hirose K, Tajima K, Rohan T, Calle EE, Heath CW Jr, et al.Alcohol, tobacco and breast cancer--collaborative reanalysis of individual data from 53 epidemiological studies, including 58,5 I5 women with breast cancer and 95,067 women without the disease. $\mathrm{Br} J$ Cancer 2002;87:1234-45.

36. Wolf I, Sadetzki S, Catane R, Karasik A, Kaufman B. Diabetes mellitus and breast cancer. Lancet Oncol 2005;6:103-II. 